\title{
Full traveltime inversion in source domain
}

\author{
Lu Liu, Aramco Beijing Research Center, Aramco Asia; Bowen Guo, King Abdullah University of Science and \\ Technology; Yi Luo, EXPEC Advanced Research Center, Saudi Aramco
}

\section{Summary}

This paper presents a new method of source-domain full traveltime inversion (FTI). The objective of this study is automatically building near-surface velocity using the early arrivals of seismic data. This method can generate the inverted velocity that can kinetically best match the reconstructed plane-wave source of early arrivals with true source in source domain. It does not require picking first arrivals for tomography, which is one of the most challenging aspects of ray-based tomographic inversion. Besides, this method does not need estimate the source wavelet, which is a necessity for receiver-domain waveequation velocity inversion. Furthermore, we applied our method on one synthetic dataset; the results show our method could generate a reasonable background velocity even when shingling first arrivals exist and could provide a good initial velocity for the conventional full waveform inversion (FWI)

\section{Introduction}

Near-surface velocity is critical for subsurface seimic imaging and reservior delineation. Conventionally, raybased tomography is used to derived the large-scale nearsurface velocity, which usually requires the picked first arrivals as input. This method may be sufficient when the veloicty is increasing with the depth and the first arrivals are clear. But when low velocity zone exist, the first arrival become shingling, which makes the first arrival very difficult to pick. So how to make the near-surface velocity building automatically and make the method work for shingling first-arrival are two challenges. Based on such consideration, we proposed the source-domain full traveltime inversion (FTI), which does not need picking the traveltime and is capable of handling the shingling first arrivals.

In this paper, we applied plane-wave full waveform inversion (FWI) on the inverted velocity generated by source-domain FTI to check whether the workflow of source-domain FTI followed by FWI is capable of generating high-resolution near-surface velocity model. FWI has showed great potential for revealing the highresolution characteristic of subsurface medium by minimizing the difference between the predicted data and the observed data. However, FWI always fails when the subsurface medium is very complex, or the initial velocity is not accurate enough or the seismic data do not have the extremly low frequencies, typically less than $2 \mathrm{~Hz}$. Therefore, many researcher used refraction or diving wave energy to implement FWI for estimating the near-surface velocity as the early-arrivals misfit function contains much fewer local minimal than the full dataset (Sheng, et al., 2006). In addition, the early arrival is more acoustic, thus the acoustic wave-equation may be sufficent to describe the wave phenomenon. For field data, the amplitude matching between predicted data and true data is very difficult to handle due to the complex physics of the subsurface and the inaccurate estimation of soure wavelet; thus we adapt the normalized cross-correlation objective function (Routh et al., 2011) to relax the requirement for amplitude matching.

In this paper, we firslty derived the adjoint source of our method; then we compare our gradients and the Born-based gradients using one simple model, that shows our gradient can distinguish the initial velocity is higher or lower and provide a right sign, but Born-based gradient can not. Furthermore, we applyied our method and FWI on one synthetic dataset, which show our method followed earlyarrival FWI can be a feasible workflow for high-resolution near-surface velocity model building.

\section{Methods}

The objective function of source-domain FTI is,

$$
E=\iiint w(x, t-p x)^{2} U(x, z=0, t ; p)^{2} d x d p d t
$$

where $p$ is ray parameter quantified by $\frac{\sin \left(\theta_{s}\right)}{v_{0}} ; \theta_{s}$ is the surface incident angle; $v_{0}$ is the surface velocity; $U(x, z=$ $0, t ; p)$ is the reconstructed plane-wave source which is calculated by backward propagating the plane-wave data via solving the acoustic equation; $w(t)$ is a Gassian window whose peak value is located at the position of true plane-wave source, which is expressed as,

$$
w(t)=1-e^{-\frac{(t-t 0)^{2}}{c^{2}}}
$$

where, $t_{0}$ is the location of true plane-wave source and $\mathrm{c}$ is the standard derivation.

When the velocity is higher than the true one, the reconstructed source will be later than the true plane-wave source; if the velocity is lower, then the reconstructed source will be earlier than the true one. When the inverted velocity approaches to the true one, the reconctructed source best focuses at the true source location and the objective fucntion reaches minimum. By minizing the time difference between the reconstructed source and the true source, we can update the velocity. 


\section{Source-domain full traveltime inversion}

The above idea was firstly proposed by Zhang and Wang (2009). Their method is sensitive to initial velocity and requires a close-to-true velocity as initial, because when the velocity has many reflector, the reconstructed plane-wave source could be very complex and make the objective fucntion fall into local minimum. Based on such consideration, here we use the early arrivals to invert the background velocity and use the plane-wave FWI to invert the high-wavenumber component of velocity.

To obtain the background velocity based on the best focus of the reconstrurcted plane-wave source, we firstly use the Born approximate to derive the gradient like Zhang and Wang (2009) did. The adjoint source is $w(x, t)^{2} U(x, z=$ $0, t)$. To verify this Born-based gradient, we applied this gradient to a $2 \times 6 \mathrm{~km}$ model which only has one flat layer. 25 plane-wave shots with a range of surface incident angle $\left[-30^{\circ}, 30^{\circ}\right]$ are used to form gradients correponding to fast and slow velocities shown in Figure $1 \mathrm{a}$ and $1 \mathrm{~b}$, respectively. It shows that the Born-based gradients fail to distinguish whether the background velocity is slower or faster than the true one. Then we apply the FTI theory (Luo et al, 2016), that assumes the velocity perturbation only causes the traveltime shifts of waveforms, on our objective function equation (1). The FTI-based adjoint source is $U(x, z=0, t) \int U(x, z=0, t)^{2} w(x, t) \frac{\partial w(x, t)}{\partial t} d t$. Using the same data and parameter, we apply the FTI-based gradient equation to the faster and lower velocity shown in Figure $1 \mathrm{c}$ and $1 \mathrm{~d}$, respectively. It clearly manifests that the FTIbased gradients have opposite signs, which correspond to the slow or fast velocities. Therefore, the conventional Born-based algorithm suffers an intrinsic problem in obtaining the correct gradient of the proposed objective function; while the FTI-based equation could overcome this sign problem.
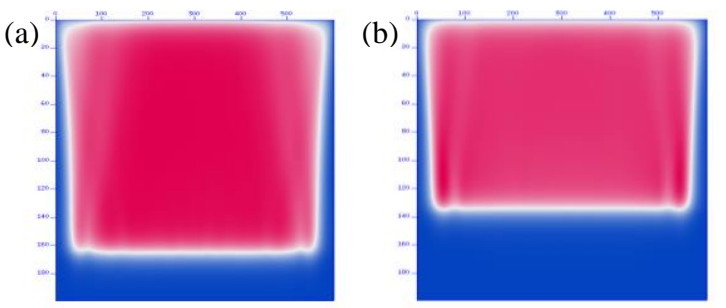

(c)

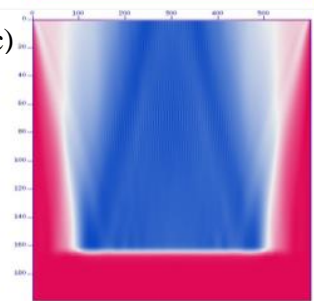

(d)

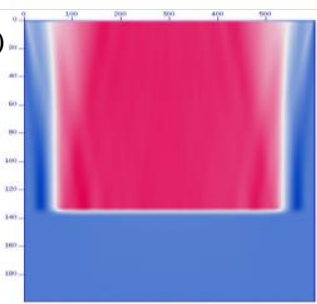

Figure 1: Compare the FTI gradient and Born-gradient for fast (10\% higher than true velocity) and low (10\% lower than true velocity) velocities using a single-layer model. (a) Born-gradient with fast velocity. (b) Born-gradient with low velocity. (c) FTI gradient with fast velocity. (d) FTI gradient with low velocity.

Besides the sign problem, the source-domain FTI method do not need picking the first arrival of data which is very time-consuming for the production of ray-based tomogrphy. Moreover, since we best focus our reconstruce plane-wave source in the source domain, there is no need to estimate the source wavelet. It is worth to mention that one-way Green function is used here to implement the wave propation. The combination of one-wave Green function and plane wave data is caple of reducing the computation cost, because the plane wave number required for our method is much smaller than the shot number and unlike the two-way Green function, the plane-wave data with oneway Green function do not need padding the trace along time direction. Besides, this method is a natural layerstripping method, because the larger surface incident angle corresponds the shallow zone, and the small angle is associated with the deep zone. Thus, we could sort the plane wave data into several groups according the surface incident angle to invert the shallow zone firstly then invert the deep part gradually. Such stratege could enhance the robustness of this method.

To further increase the quality of the near-surface velocicy model, we applied FWI on the output velocity of sourcedomain FTI. In order to improve the efficiency and relax the amplitude sensitivity, we used normalized crosscorrelation objective function with plane-wave data as input. The objecitve function is written as,

$$
E=\sum_{p=1}^{n_{p}} \sum_{x_{g}=1}^{n_{g}} \frac{\sum_{t} d_{o b s}\left(t, x_{g}, p\right) d_{p r e}\left(t, x_{g}, p\right)}{\left\|d_{o b s}\left(t, x_{g}, p\right)\right\|_{2}\left\|d_{p r e}\left(t, x_{g}, p\right)\right\|_{2}}
$$

Where, $d_{o b s}\left(t, x_{g}, p\right)$ and $d_{\text {pre }}\left(t, x_{g}, p\right)$ are the observed and predicted plane-wave data at the location of receiver $x_{g}$ corresponding to the ray parameter of $p$, respectively; $n_{p}$ and $n_{g}$ are the number of plane-wave data and recivers, respectively.

\section{Numerical Examples}

The algorithm described above has been applied on a 2D wedge model (shown in Figure 2a) whose size is $2.4 \times 18$ $\mathrm{km}$. Such model with low velocity zone will generate the shingling first arrival, which is difficult for first-arrival picking of ray-based tomography. We use a Ricker wavelet with the peak frequency of $12 \mathrm{~Hz}$ to generate 600 shots via one-way wave-equation Born modeling. There are 600 receivers for each shot with spacing of $30 \mathrm{~m}$ covering the entire model at the surface. The maximum recording time is $5.0 \mathrm{~s}$, and the time sampling interval is $4 \mathrm{~ms}$. Here we only used 40 plane-wave data with a surface incident angle range $\left[-63^{\circ},-23^{\circ}\right]$ and $\left[23^{\circ}, 63^{\circ}\right]$ for inversion. The initial 


\section{Source-domain full traveltime inversion}

model for the source-domain FTI is a linear velocity from $1500 \mathrm{~m} / \mathrm{s}$ to $4500 \mathrm{~m} / \mathrm{s}$.

Figure $2 b$ is the inverted velocity obtained by sourcedomain FTI, which shows the large-scale velocity structrue has been well inverted. To check the quality of the result, we overlapped the true plane-wave source (red dashed line in Figure $2 \mathrm{c}$ and $2 \mathrm{~d}$ ) of surface incident angle $43^{\circ}$ with the initial and the inverted reconstructed source, which is shown in Figure 2c and 2d, respecctively. Figure $2 d$ shows the reconstructed plane-wave source using inverted velocity matches with the true one very well.

Then we applied the plane-wave FWI on the result of source-domain FTI. The result is shown in Figure 3a, which clearly show that the final inverted result is very close to the true one. Then we implement twice forward modeling using true model and the final inverted model, and the two datasets are displayed in Figure $2 b$ together for the convience of comparison. It manifest that the predicted data matches very well with the observed data. Thus, the source-domain FTI method is capable of producing good initial velocities for FWI, and the workflow of sourcedomain FTI plus FWI could be used for the near-surface velocity model building.
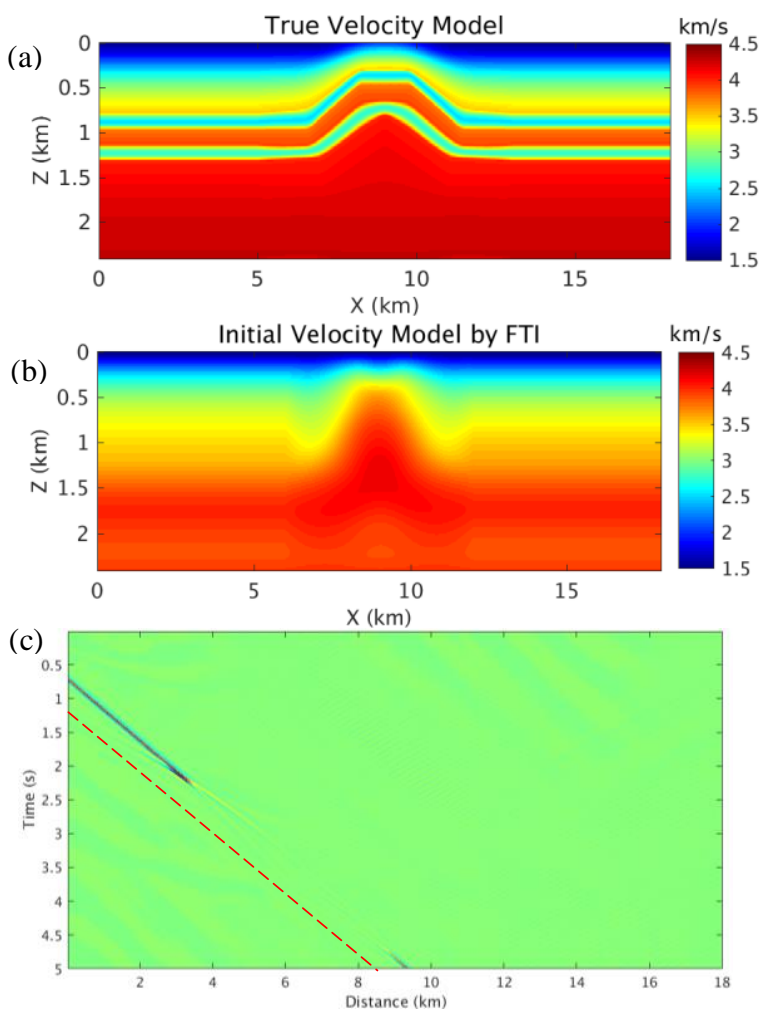

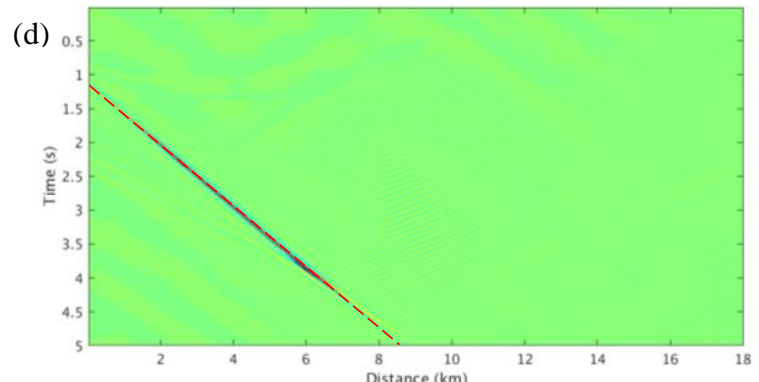

Figure 2. Results of source-domain FTI on a 2D wedge model. (a) True velocity model. (b) Inverted velocity via source-domain FTI with a linear velocity as an initial model. (c) Reconstructed planewave source using the initial model overlapped with the position of true plane-wave source (red dashed line). (d) Reconstructed planewave source using the inverted velocity overlapped with the position of true plane-wave source (red dashed line).
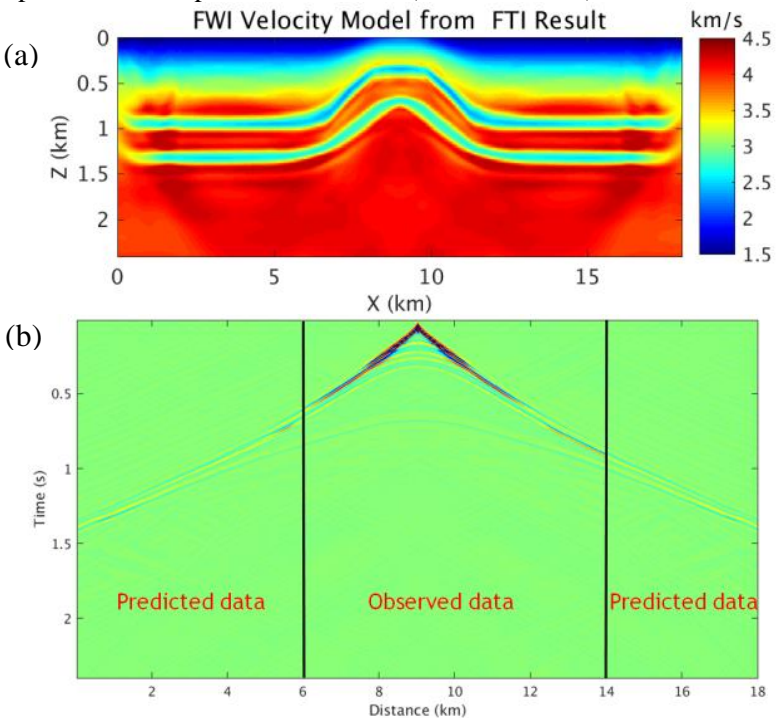

Figure 3. Results of plane-wave FWI. (a) Inverted velocity obtained by FWI with the result of source-domain FTI as initial. (b) Data comparison between observed data and predicted data using the final inverted velocity.

\section{Conclusions}

We proposed the source-domain full traveltime inversion to invert a source-domain kinematically accurate velocity. Unlike the traditional ray-based first-arrival tomography, this method is automatic and do not require picking the first arrival of seismic data as input for the inversion. Besides, this method do not need estimate the source wavelet. Moreover, this method overcomes an intrinct problem of Born-based gradient, that is it can provide the right sign for the higher or lower initial velocities. The synthetic test demonstrate that our method followd by FWI could be a effective tool for near surface veloicty model building. 


\section{Source-domain full traveltime inversion}

\section{Acknowledgements}

The authors would like to thank Saudi Arabian Oil
Company (Saudi Aramco) for its support and permission to publish this paper. We also thank our colleagues Yan $\mathrm{Wu}$ and Yue Ma for many useful discussions.

\section{References}

Luo, Y., Ma, Y., Wu, Y., Liu, H. and Cao, L., 2016. Full-traveltime inversion. Geophysics, 81, no. 5, R261-R274, doi: 10.1190/geo2015-0353.1

Routh, P.S., Krebs, J.R., Lazaratos, S., Baumstein, A.I., Chikichev, I., Lee, S., Downey, N., Hinkley, D. and Anderson, J.E., 2011. Full-wavefield inversion of marine streamer data with the encoded simultaneous source method. 73rd Conference and Exhibition, EAGE, Extended Abstracts, F032.

Sheng, J., Leeds, A., Buddensiek, M. and Schuster, G.T., 2006. Early arrival waveform tomography on near-surface refraction data. Geophysics, 71, no. 4, U47-U57, doi: 10.1190/1.2210969

Zhang, Y. and Wang, D., 2009. Traveltime information-based wave-equation inversion. Geophysics, 74, no. 6, WCC27-WCC36, doi: 10.1190/1.3243073

(OThe Society of Exploration Geophysicists and the Chinese Geophysical Society

2017 CGS/SEG International Geophysical Conference

Qingdao, China, 17-20 April 2017 